\title{
The Implementation of Management Rights for Ethereum Forking Governance
}

\author{
WAI WAI WONG \\ Faculty of Business, Communication and Law, INTI INTERNATIONAL UNIVERSITY, MALAYSIA. \\ E-mail: Waiwai.wong@newinti.edu.my
}

\begin{abstract}
Ethereum is an open-source, decentralized applications to support a cryptocurrency-based platform. The recent Ethereum hard fork, called 'Istanbul' took place in October 2019 due to threats from multitude of competitors has been completed. This hard fork brings six Ethereum Improvement Proposals (EIPs) which includes code execution, blockchain's mining algorithm, increase data storage process capacity and also reduce Gas costs. Although these changes intended to improve the performance of Ethereum blockchain, many people have raise concern that forking is controversial. Cryptocurrency forks usually brings big changes ahead and may have serious implication on the price of Ethereum. Many resource appropriators (coin holders) are anxious for next network update for Ethereum. Most of the rules and decisions are made before these participants join the system or leave in the hands of developers of the project. There is lack of effective means for most resource appropriators or stakeholders to participate in system of decision-making for the next network update. This article provides a comprehensive overview of how decision-making for network update to be made in Ethereum and to propose the implementation of the management rights concerning the design, implementation of future changes to the Ethereum platform in order to achieve a clear collective-choice arrangement that allow most resource appropriators and stakeholders to participate in the decision-making process within the Ethereum community.
\end{abstract}

Keywords: Ethereum Forking, Management Rights, Decision-Making, Collective Choice

JEL Classification: O3, K3

Recibido: 9 de Febrero de 2021

Aceptado: 2 de Marzo de 2021 


\section{Introduction}

Ethereum is an open-source, decentralized applications to support cryptocurrency-based platform. The recent Ethereum hard fork, called 'Istanbul' took place in October 2019 due to threats from multitude of competitors has been completed. Although the hard fork intended to improve the performance of Ethereum blockchain, many people have raise concern that forking is controversial. Many resource appropriators (coin holders) are anxious when will be the next network upgrade or forking for Ethereum. There is lack of effective means for most resource appropriators or stakeholders to participate in system of decision-making for the next network upgrade. The objectives of this article are to provide a comprehensive overview of how decision-making for network upgrade to be made in Ethereum and to propose the implementation of the management rights concerning the design, implementation of future changes to the Ethereum platform. This article will be divided into three parts. Part I will explain forking, the latest Ethereum forking took place in 2019. Part II will provide an overview as to how decision-making for network upgrade to be made and its implications. Finally, Part III this article proposes the implementation of management rights to resource appropriators in order to allow them to participate in the decision-making process within the Ethereum community.

\section{Ethereum Forking}

Ethereum is the foundation for a new era of the internet. It is a global, decentralised, blockchainbased platform that facilitate the operation of smart contract. With smart contract, it do away with downtime, fraud and minimize third party interference. Anyone can execute a smart contract through this open-access infrastructure, controlled by no company or decentralised body. (S. Asharaf, S. Adarsh, 2017) Smart contract further enables business to create new markets, to raise funds, to keep registries of debts or promises and/or transfer funds according to instructions that has been stipulated without the middle man and any counterparty.(Petersson,D., 2018).

Apart from creating endless opportunities, Ethereum platform spares users of coding when one wants to create and execute smart contract. (Friebe, T. 2017) Ethereum platform provides template that deploys smart contract with various functions such as configurable messages and create fungible token.(Hayes, A., 2020) With that, users can create smart contract more quickly and apply modification with ease. This is one of the factors contributing to the ongoing growth of Ethereum. In 2016, Ethereum conducted a radical experiment called the Distributed Autonomous Organization (DAO) which created a venture capital firm that made decision through smart contract. This DAO is like corporations, engaging in various economic transactions such as buying and selling things, negotiating deals, balancing budgets and even hiring labour without any human or institutional intervention.(Hayes,A., 2020).

However, in 2016, hacker found a loophole in the coding of one of the applications build on Ethereum that allowed him to drain funds from the DAO. The attacker was able to ask the smart contract DAO to give Ether multiple times before the smart contract could upgrade the balance. Ethereum platform was hacked and resulted in $\$ 50$ million worth of funds were stolen. Vitalik Buterin, the founder of Ethereum proposed a 'soft fork' that would prevent the attacker to make the transactions valid. The stolen funds are locked in a "Child DAO" and are unable to be moved for 28 days. It invalidated all the illegal transactions. Later 'hard fork' was created in order to change the network's protocol. Once a hard fork has been implemented, it requires all nodes or users to upgrade to the newest version that would create a permanent divergence from the previous version. By doing that, they were able to return the stolen funds to the original owners, as per the records prior to the hack.

As we can see in the 2016 incident, soft fork was being proposed in order to freeze all further movement of funds. However, the soft forks could introduce denial of service attack which would prevent the network from processing valid transaction. The attacker can execute difficult computation and flood the network with malicious transactions.(Hess, T., 2016) Due to this possible flaw, hard fork was proposed to create a new version of the network with different protocol. It brings major change 
to the network in question. Hard fork further changes the cryptocurrency protocol and render the old version of the protocol invalid. However, in the case of Ethereum, the old version continues, it resulted in the split from the new version.

Other major upgrades on Ethereum are EtherZero and Metropolis. Ether Zero is another wellknown Ethereum fork with some of the key features such as improving the transaction speeds, zero transaction fees and implementation of a Proof of Stake consensus algorithm that is contingent upon 'masternodes', or 'voters' that approve the blocks on the chain that within the Ethereum network.(Aziz, n.d.) The current Ethereum fork is Metropolis. Metropolis is to improve the existing Ethereum blockchain. It consists of three stages namely, Byzantium, Constantinople and Serenity finally. In the phrase of Byzantium, there were a total of nine different Ethereum improvement protocols (EIP) that aims to enhance scalability, security and privacy.(Aziz, n.d.) In the phrase of Constantinople which is divided into 2 stages.(Foley, 2019) The first stage, St. Petersburg, made it cheaper to do certain things on-chain. The second stage of Constantinople called Istanbul, introduces more privacy, scaling capabilities and rebalancing of the gas pricing with the computation costs of the EVM opcodes. Istanbul further divided into 2 stages: Muir Glacier and Berlin. The Muir Glacier update was on Jan. 2, 2020, with only one improvement proposal that aims to delay the difficulty bomb, a built-in algorithm of the Ethereum blockchain that could significantly increase the difficulty in mining a new block.(Cacioli, 2019) Berlin hard fork tentatively scheduled in June 2020, includes changes on ProgPoW (EIP-1057), for improving GPU-mining, EIP-1380, EIP-2045, EIP-2046 connected with gas costs and EIP-1985 responsible for the optimization of the virtual machine. Once this phrase is completed, the Ethereum blockchain will be ready for the long-anticipated implementation of 'Serenity', the protocol upgrade formalize to switch to Proof of Stake.(Aziz, n.d.).

\section{Overview of Decision-Making for Network Upgrade and its Implications}

"The Hard Fork is a delicate topic and the way we see it, no decision is the right one. As this is not a decision that can be made by the foundation or by any other single entity, we again turn towards the community to access its wishes in order to provide the most appropriate protocol change." Stated by Ethereum Cofounder Jeffrey Wilcox.

The 2016 Ethereum hard fork was implemented to restore all the crypto coins that have been stolen, however many people did not agree with the hard fork because they see this as a violation of the fundamental meaning of 'decentralized'. A truly decentralized cryptocurrency means no interference by the developers whatsoever. Once the developers take action, this would cause implication on the cryptocurrency in the future. Therefore, in decentralized platform like Etherreum, the Ethereum community acts as decision maker. The Ethereum community refers to the group of people that includes institutions, companies and other organizations that support and maintain the Ethereum blockchain.(Friebe, 2017) However, within this community, core developers have great influence over the others and they are trusted by miners to provide technical guidance. For example, in 2016, core developers were in favour for hard fork and most of the miners followed their decision. Minority of miners did not support this idea and continued mining the old Ethereum blockchain which resulted in a split. This incident shown decision making power distribution on the Ethereum blockchain essentially lies in the hands of core developers.

The introduction of Ethereum Improvement Proposals (EIPs) is a design document providing information to the Ethereum Community on deciding a new feature for Ethereum, its process or environment.(Ethereum Improvement Proposals, n.d.) The EIPs process involves several stages. Any person, whether developer, miner, full node operator, or any other stakeholder are allowed to participate to EIPs. First, the person who propose changes, called champion or EIP author vet his/her idea in the respective forums such as the Ethereum Magicians forum or Ethereum subreddit.(Ethereum Improvement Proposals, n.d.) Secondly, in Core EIP, it allows client implementers to discuss the technical merits, gauge what other clients will be implementing and to coordinate EIP implementation for network upgrades.(Ethereum Improvement Proposals, n.d.) Thirdly, the champion write a draft in a 
Github pull-request and the EIP process includes status of idea, draft, last call, accepted and final. A team of EIP collaborators will administers the process and maintains the status of EIPs. For an EIP to get a final pass, it needs to be accepted by the community, especially by miners.

This entire process brings some structure to Ethereum forking governance. However, there are three main points to be addressed here. First is on the issue of dominance. As we can see, the entire process is dominant by developers as they have strong influence over the miners and the rest of the community. Many stakeholders lack the technical knowledge and understanding and hence they will just follow the recommendations made by the developers. This was evidenced in 2016 when Vitalik Buterin implemented a hard fork in order to reimburse monies back to investors. Many see Buterin's role as "benevolent dictator". He persuaded the majority to follow his solution and do the upgrades. Similar situation occurred during the early days of Bitcoin when control of the protocol rested in hands of Satoshi. Later, the Bitcoin Core developers were responsible for the majority of development on the Bitcoin protocol and hence created the perception that Bitcoin Core developers had an authoritarian influence over the protocol's development in Bitcoin(Clarke, 2019; Qureshi, Muhammad Imran, et al. 2020).

Secondly is on the issue of decentralization. Some people see when a small group of developers have strong influence over the decision, it is less democratic and is a violation of the fundamental meaning of 'decentralized' which controlled by no company or decentralised body. If smart contracts held to be inviolable but can be overturned by a collective decision of the community, how can they guarantee immutability in their record or transaction? However, on the other hand, centralized controlled leads to quicker decision making on protocol improvements. Thus decisions about protocol changes are made more efficiently such as the immutability of transactions. A more centralized governance approach might be better for developers of decentralized applications (Dapps) who depend on Ethereum's ability to solve scalability challenges.

Thirdly is on the issue of other factors. Apart from the evaluation on the technical aspect of implementing and executing new features as proposed in EIPs, there are other non-technical factors which are crucial to decide on the implementation of hard fork. The EIPs process and AllCoreDevs call were not designed to address contentious non-technical issues which may become hinders to hard fork implementation. Contentious non-technical issues such as what are the criteria for participating in a fork event; product and service impact; tax implication; market risk and liquidity demand. It is also important to take note that organizations may choose to retain the right to determine which fork to be used with reference to currency for portfolio pricing. For example, in October of 2017, Bitcoin Gold was created as a result of a hard fork from Bitcoin. There was general disagreement and concern about the technology behind Bitcoin Gold and potential vulnerabilities. Because of this, Bitcoin Gold was not recognised in many cryptoasset exchanges such as Coinbase, Poloniex and Kraken.(O'Neal, 2018).

\section{The Implementation of Management Rights to Resource Appropriators}

One of the biggest concerns of the investors is when will be the next forking occurs. It is because forking can have a significant impact on the price of Ethereum. Back in 2016 under the DAO attacked, the price of Ethereum reacted violently, falling from an all-time high of $\$ 21.52$ on 17th June to $\$ 9.96$ the following day. However, on the other hand, when Constantinople hard fork was implemented in 2019, the Ethereum community and most exchanges supported the fork and therefore the price did not react the same way as in 2016 because it was not a contentious hard fork.(Olszewicz, 2019) Investors or Ethereum users do not want to see huge volatility in the price of Ethereum. In order to prevent volatility in relation to implementation of forking, we need to have a consistent framework and strong governance for future forking or network upgrade. Such consistent framework and strong governance must be able to addressed the three main points - dominance, decentralization and other factors (non-technical) as stated in Part II of the discussion in relation to decision-making for network upgrade for Ethereum.

Network upgrade not only introduce technical upgrade but also important economic and ethical considerations. These considerations often been overlooked among the community of blockchain 
developers.(A, n.d.) In formulating the appropriate framework with strong governance, the elements of economic and ethnic should be part of the framework and governance. Reference may be made to the proposed governance framework recommended by Tae Wan kim and Ariel Zetlin-Jones.(A, n.d.) The proposed framework permit change or class of changes in protocol. The proposed framework should consist of the following fixed features:

\subsection{Substantive Principles}

1. Utility-Enhancement Principle: The change in the protocol maximizes (or least enhances) the overall value of the cryptocurrency in question.

2. Generalization/fairness Principle: The change is something that everyone is allowed to do in similar circumstances, if they can.

\subsection{Procedural Principles}

1. Publicity Principle: Decisions and rationales behind the decisions must be publicly accessible to all coin users.

2. Revision and Appeals Principle: There must be mechanisms for challenge and dispute resolution regarding decisions, and, more broadly, opportunities for revision and improvement of policies in light of new evidence or arguments.

3. Regulation Principle: There is either voluntary or public regulation of the process to ensure that conditions $1-4$ are met.

Utility is basically an intrinsically valuable principle to ensure common good and make gain financially.(Hooker, J. N., and Williams, 2012) Utility principle is a necessary condition for all proposal.(Hooker, J. N., and Williams, 2012) Therefore, in the context of Ethereum or cryptocurrency in general, the proposal for network upgrade must be able to increase the value of the crypto in question. On the other hand, the condition of generalization is a well-established ethical test to secure fairness.(Hooker, J. N., and Kim, 2018) For instance, in deciding whether a network upgrade should be implemented, such upgrade must be a decision that every similar size group is allowed to do and justified. The reason to justify this particular upgrade, the same reason should also justify for anyone to whom the reasons apply.(A, n.d.) The proposed framework consists of these two fundamental principles would safeguard overall value and fairness in the system.

Under the procedural aspect, the publicly principle is very essential because it ensure transparency in decision-making process. It further allowed the proposer of the network upgrade to be scrutinized by the various stakeholders of the cryptocurrency. The appropriate framework with strong governance must have mechanism relate to revision and appeal principles to the proposal or decision. With the mechanism of revision and appeal, if one contest on the issue under the substantive aspect, this mechanism would allow the proposer to offer an explanation as to why it should be done. Other stakeholders can also provide their view and different perspective on the proposed network upgrade. Both of these two principles would lower the degree of dominance by developers and take into consideration the non-technical factors such as product and service impact; tax implication; market risk and liquidity demand that has been explained earlier in Part II of this article. A regulatory framework should be in place but it should be in the form of policy rather than strict law in governing implementation of Ethereum forking (Qureshi et al., 2020).

Network upgrade introduce important economic and ethical considerations that we believe have not been considered among the community of blockchain developers. (A, 2019) Hence, this article suggests that the process for Ethereum Improvement Proposal (EIP) should follow the phrases:

1. The person who proposed network upgrade, the EIP author can vet his/her idea in the respective forums. This would allow the EIP author to get the general sentiment of the propose changes from the Ethereum community. 
2. Secondly, in Core EIP, it allows client implementers to discuss the technical merits and to see what other clients have done in a similar situation. This apply the generalization principles.

3. Thirdly, the EIP author write a draft in a Github pull-request and the EIP process includes status of idea, draft, last call, accepted and final. In this stage, most of the technical issues will be already dealt with and settled.

This is the additional stage this article proposed to the existing EIPs that incorporate the substantive and procedural principles:

4. The EIP author organise a forum, such forum can be held physically or virtually to present his/her idea to the various stakeholders:

(a) The EIP author should draft his/her idea of the proposed network upgrade similar to Whitepaper for Initial Coin Offer, a document which outline everything investors or stakeholders they need to know about the proposed network upgrade such as benefit, possible financially gain, improvements, team, road map, timeline for implementation, backup and future plan. Furthermore, the draft should explain whether the proposed network upgrade is consistent with the initial whitepaper and any departure from it must be explained in details.

(b) EIP author set the agenda for such forum and discuss the various factors in relation to the proposed network upgrade.

(c) Depend on the complexity of the proposed network upgrade involves, various committees should be appointed by the stakeholders to examine specific aspect in relation to the implementation of network upgrade. For example, there should be a committee dealing for taxation; product and service impacts; operational impact; market risk; implication on prices; liquidity issues and support from exchanges. Each committee should submit their report base on their findings and the report should be made publicly available to all investors and stakeholders.

(d) At the revision, amendments should be made base on the findings and recommendations of the committees. Appeal will be conducted to allow the EIP author, his team or any party to offer explanation on proposed network upgrade. This will enhance communication among the parties, gain more consensus and legitimacy.

(e) Finally, voting would decide whether the proposed network upgrade should be implemented or not. Each stakeholder should have the equal right to vote and proper voting procedure should be established.

Voting is always regarded as an important right and a mechanism for stakeholders to participate in the decision- making of forking or network upgrade. The use of on-chain votes has been used in Bitcoin and Ethereum to avoid over governance.(Kim, 2019) Each token or cryptocurrency holder, by virtue of his/her ownership, have a say in the fortunes of a network's technological progress.(Kim, 2019) However this does not represent the true picture. According to Santi Siri, the founder of non-profit Democracy Earth, he stated that the problem with Blockchain voting today is that it is $100 \%$ plutocratic. Despite the voting system in place to enhance consensus, the decision-making still be dominated by a small number of people who has the technical knowledge and power. Siri further stated that:

"It's based on whoever has the largest amount of tokens or the largest economic weight.

... [Token] holders don't have any weight at all in the decision-making. The voting is pretty much irrelevant if a single whale can decide the outcome of an election."(Kim, 2019)

For example, there were $65 \%$ of miners voted against the Ethereum hard fork in 2016, however, the announcement came from the Ethereum community that the vote would be overturned and the hard fork would still be carried out.(Subery, n.d.) This incident showed the vulnerability of voting system despite the fact that voting is regarded as a core value proposition for the Ethereum blockchain.

There are still lot of issues yet to be resolved in the Ethereum voting protocol. The first issue is counting of vote. The Carbonvote was initiated during the DAO hard fork and it was used to conducted 
vote in a secure web-page.(Carbonvote, n.d.) It had become a great source of reference when the community decided to go with the fork. It has a choice of motion in which allow the stakeholders to vote and show of preference. However, in respect of counting, Carbonvote count coins, not individual. How many ethers a stakeholder have, that how many votes he can cast. Therefore, those who owns large numbers of Ether wold eventually dominate this so called 'democratic' consensus. There is also a possibility of double voting. For example, when $A$ cast a vote, subsequently $A$ sold his Ether to $B$ and $B$ cast another vote base on the same amount of Ether. It does not matter whether $A$ and $B$ are the same person or vote the same way, the system does not recognised the notion of an individual and this double voting is a problem.

Perhaps one may conclude the most effective way of governance does not lie in voting but rather establish a true transparency and governance based on the social interaction among the stakeholders and the organisation. This proposition is supported by CEO of developer group Aragon One Luis Iván Cuende. Santi Siri, the founder of non-profit Democracy Earth stated that:

"When it comes to the promise of blockchain in relation to governance, it is a very important promise," Siri emphasized. "The ability to inspect the interactions that happen in an election [or vote] in a permission-less way brings tremendous amount of transparency to the process of having more fair governance."(Kim, 2019)

The next question is how do we ensure more interaction among the stakeholders in the process. Now we turn to look at Schlager and Ostrom bundle of property rights includes access to resources, withdraw from the resources, management, exclusion and ailenation.(Schlager \& Ostrom, 2010) Out of these five rights, the right in management is relevant in order to ensure more interaction in the process and to enhance true transparency and governance based on the social interaction among the stakeholders and the organisation. According to Schlager-Ostrom framework, management means actions which change the flow of goods and services associated with the resource. Management rights are oriented towards improving or enhancing the resource. The emphasis under the management right in the Schlager-Ostrom framework is that management right is a continuum, sub-categories arrayed around the extent of transformation or diminishment, with alteration throughout the process. (Rozas, David and Tenorio-Fornés, Antonio and Díaz-Molina, Silvia and Hassan, 2015) To apply this in the Ethereum forking governance, the management right should not only be exercised at the time when forking decision needs to be made. A successful management requires not only a collective choice in decision-making at that time but also monitoring the progress of upgrades and adjustment to that decision or process if necessary to ensure the success of forking implementation. The proposed possible financially gain, improvements, road map, timeline for implementation, backup and future plan all should be monitored accordingly and the EIP author should be held accountable for the progress and success to the rest of the community and stakeholders.

This article further proposed that after the Ethereum network upgrade, EIP author or whoever responsible to this network upgrade should further conduct post fork valuation meeting to allow the organisation to report on the progress of the network upgrade. The proposed post fork valuation should not only look into the technical aspect of implementation but also the impact on the other aspects such as taxation; product and service impacts; market risk; operational risks; implication on prices; liquidity issues and support from exchanges which has been discussed during the EIP author organised initial meeting on the proposed network upgrade. The valuation meeting is a forum for parties to have interaction, to jointly evaluate and make further improvement on the proposed network upgrade.

\subsection{Impact}

The proposed additional process to be incorporated to the Ethereum Improvement Proposal (EIP) and the implementation of management right exploring the relationships between technical and social power within the Ethereum eco system. The collaboration in the process and management after the network upgrade is for the purpose of introducing decentralisation of power over the forking 
governance structure.(Rozas, David and Tenorio-Fornés, Antonio and Díaz-Molina, Silvia and Hassan, 2015) These organisational changes entail constant negotiation and interaction among the members of the eco system. Negotiations and interaction can be understood as part of the generation of collective-choice arrangements which will make the decision that derived from there are more receptive and legitimate. This generation of collective-choice arrangements will ensure more equality of power as opposed to authoritarian approach.

The proposal would also increase the level of transparency and to open the organisational processes to the greater community. It always has been a long tradition in decentralized technology that to make processes as open and participative as possible. This strong culture of openness and participation will be further enhanced if one truly implements the management rights according to the SchlagerOstrom's principles in the Ethereum community. This would provide means of accountability and increase the legitimacy of these processes. (Rozas, David and Tenorio-Fornés, Antonio and Díaz-Molina, Silvia and Hassan, 2015)

Furthermore, implementation of management right will encourage stakeholders to get themselves familiar with the operation of the decentralized network. There are good portion of investors out there with little knowledge on how the decentralised network works. They invest in cryptocurrency due to herd mentality, which basically follow and copy the decision of the others.(CFI, n.d.) With the implementation of management right, it encourage greater participation and culture in collective decision-making in the cryptocurrency community. Investors with insufficient knowledge in this respect would find themselves out of place. Two possible outcomes would occur from this: (1) Investor may choose to move on to other investment; or (2) Investor may try to familiarise himself/herself in order to make an informed decision. This would create positive impact because by removing those investors has little knowledge on decentralized network can possibly remove volatility and price spikes due to herd mentality.(Xuan Vinh Vo, 2019) Good investors analyse the growth pattern and how the company or organisation operates. They would research into the company strategy and philosophy and overall market sentiment. With this knowledge, it would significantly contribute to the effectiveness in forking governance in decentralized community.

\section{Conclusion}

Ethereum forking is considered as an important event among Ethereum community and its network upgrade is to improve the existing protocol generally making Ethereum cheaper, faster and more efficient. The decision-making on Ethereum forking largely lies in the hands of the developers which often resulted in over dominance, go against decentralization policy and overlook other non-technical factors. To resolve this and to establish an appropriate framework with strong governance on Ethereum forking, this article proposed an additional stage - conduct forum discussion to the existing EIPs is necessary to enhance interaction between various stakeholders and the organisation. The implementation of management right is an ongoing right and responsibility that not only encourage various stakeholders or resources appropriators to make collective choice in decision-making at that time of implementation of fork but also monitoring the progress of upgrades and adjustment to that decision. With greater interaction between the various stakeholders and the organisation, that would increase the level of transparency and opening the organisational processes to the greater community, hence achieve towards decentralization of power and self-governance.

\section{References}

1. A, K. T. And Z.-J. (2019). "The Ethics of Contentious Hard Forks in Blockchain Networks With Fixed Features." https://doi.org/10.3389/fbloc.2019.00009

2. Adam Hayes (2020):Is Ethereum more Important than Bitcoin? 24 January, https://www.investopedia.com/articles/investing/032216/ethereum-more-important-bitcoin.asp

(Date of Access: 23/02/2021) 
3. Aziz. (N.D.): Guide To Ethereum Hard Forks: Ethereum Classic, Etherzero \& Metropolis. https://masterthecrypto.com/ethereum-hard-forks-guide-ethereum-classic-etherzerometropolis/ (Date of Access: 23/02/2021)

4. Cacioli, L. (2019): Ethereum - The Whole Forking History. December 8, https://blockchain.news/news/ethereum-the-whole-forking-history (Date of Access: 23/02/2021)

5. Carbonvote. (N.D.), http://carbonvote.com/ (Date of accessed: 23/02/2021)

6. CFI. (n.d.). What is Herd Bias? https://corporatefinanceinstitute.com/resources/knowledge/trading-investing/herd-mentalitybias/\#: :text=What\%20is\%20Herd\%20Mentality\%20Bias,by\%20their\%20own\%20independent\%2 Oanalysis.\&text=|t\%20focuses\%20on\%20the\%20fact\%20that\%20investors\%20are\%20not\%20alwa ys\%20rational. (Date of Access: 23/02/2021)

7. David Clark (2019): Bitcoin Governance: What are BIPs and how do they work? April 16, https://www.sfox.com/blog/bitcoin-governance-what-are-bips-and-how-do-they-work/(Date of Access: 23/02/2021)

8. David Petersson. (2018): How Smart Contracts Started And Where They Are Heading." October 24, https://www.forbes.com/sites/davidpetersson/2018/10/24/how-smart-contracts-started-andwhere-they-are-heading/?sh=475e56a637b6 (Date of Access: 23/02/2021)

9. Maxwell Foley. (2019): Ethereum's Istanbul Hard Fork Important Updates, Explained. https://cointelegraph.com/magazine/ethereum-hard-fork-istanbul-2019/(Date of Access: 23/02/2021)

10.Ethereum Improvement Proposals, https://beincrypto.com/ethereum-eip-1559-developmentprogresses-gas-crisis/ (Date of Access: 23/02/2021)

11.Friebe, T. (2017): Ethereum Governed by Benevolent Dictator? October 31, Get started building on Ethereum. https://medium.com/blockchainspace/ethereum-governed-by-a-benevolent-dictator2a2be8aa331a (Date of Access: 23/02/2021)

12.Hooker, J. N., And Kim, T. W. N. (2018) "Toward non-intuition-based machine and artificial intelligence ethics: a deontological approach based on modal logic" AIES '18: Proceedings of the 2018 AAAI/ACM Conference on Al, Ethics, and Society, Pages 130-136 https://doi.org/10.1145/3278721.3278753

13.Hooker, J. N., And Williams, H. P. (2012). "Combining equity and utilitarianism in a mathematical programming model." Manag. Sci. 58, 1682-1693. https://doi.org/10.1287/mnsc.1120.1515

14.Christine Kim, (2019): How Blockchain Voting Supposed To Work (But in Practice It Rarely Does),. (n.d.). June 8, 2019. https://www.coindesk.com/how-blockchain-voting-is-supposed-to-work-butin-practice-rarely-does (Date of Access: 23/02/2021)

15.O'neal, S. (2018): Why Bitoin Gold Got Delisted From BitTrex? September 5, https://cointelegraph.com/news/why-bitcoin-gold-got-delisted-from-bittrex (Date of Access: 23/02/2021)

16.Olszewicz, J. (2019): Ethereum Price Analysis - Constantinople hard fork imminent. 11 January, https://bravenewcoin.com/insights/ethereum-price-analysis-istanbul-hard-fork-scheduled-fornext-week (Date of Access: 23/02/2021)

17.Qureshi, M. I., Khan, N., Ahmad Hassan Gillani, S. M., \& Raza, H. (2020). "A systematic review of past decade of mobile learning: What we learned and where to go." International Journal of Interactive Mobile Technologies, 14(6), 67-81. https://doi.org/10.3991/IJIM.V14I06.13479.

18.Qureshi, Muhammad Imran, et al. "Classifications of sustainable manufacturing practices in ASEAN region: A systematic review and bibliometric analysis of the past decade of research." Sustainability 12.21 (2020): 8950.

19.Rozas, David And Tenorio-Fornés, Antonio And Díaz-Molina, Silvia And Hassan, S. (2015): When Ostrom Meets Blockchain: Exploring the Potentials of Blockchain for Commons Governance, 
https://medium.com/@eraser/when-ostrom-meets-blockchain-exploring-the-potentials-ofblockchain-for-commons-governance-413ce7ac0fcd (Date of Access: 23/02/2021), https://www.coindesk.com/ethereums-istanbul-hard-fork-is-now-live (Date of Access: 23/02/2021)

20.S. Asharaf, S. Adarsh. (2017). "Decentralized Computing Using Blockchain Technologies and Smart Contracts: Emerging Research and Opportunities." Advances In Information Security, Privacy, and Ethnics, ISBN-10: 1522521933

21.Schlager, E., \& Ostrom, E. (2010). "Property-Rights Regimes and Natural Resources: A Conceptual Analysis Property-Rights Regimes and Natural Resources: A Conceptual Analysis Edella Schlager and Elinor Ostrom property rights and the rules used to create." Land Economics, 68(3), 249-262.

22.Subery, W. (N.D.): Ethereum Hard Fork Due Date, Miner voted Overturned, https://news.bitcoin.com/ethereum-hard-fork-miner-vote-overturned/(Date of Access: 23/02/2021)

23.Tjaden Hess, R. K. And E. G. S. (2016): Ethereum's DAO Wars Soft Fork Is A Potential Dos Vector. June 2016. https://hackingdistributed.com/2016/06/28/ethereum-soft-fork-dos-vector/(Date of Access: 23/02/2021)

24.Xuan Vinh Vo, D. B. A. P. (2019). "Herd behavior and idiosyncratic volatility in a frontier market." Pacific-Basin Finance Journal, Volume 53, 321-330. 